\title{
Etude De L’impact De La Consanguinité Sur La Santé Des Descendants Dans La Population De Tiflet (Maroc)
}

\author{
Abbad Zhour \\ Drissi Ahmed \\ Abdelmajid Soulaymani \\ Khadmaoui Abderrazak
}

Laboratoire de Génétique et Biométrie, Département de Biologie,

Faculté des Sciences, Université Ibn Tofail, Kénitra, Maroc

\section{Oukarroum Abdallah}

Département de Chimie et Biochimie, Université de Québec à Montréal, Succ. Centre-Ville, Montréal, Québec, Canada

doi: 10.19044/esj.2016.v12n15p143 URL:http://dx.doi.org/10.19044/esj.2016.v12n15p143

\begin{abstract}
Several studies confirm the implication of the consanguinity in health problems and some countries still preserve this marital behavior. In this study, our objective is the analysis of consanguinity's impact on descendants' health in the Tiflet city and its regions (Northwest of Morocco). Data was collected between June and November 2012 and it was conducted on 1000 families. The calculation of the rate of the mortality concerned 3345 alive born descendants, while the biological effects of the consanguinity study was made on 3241 descendants. The results of this study show a significant association between the consanguinity and its incidence on health, such as the morbidity, the mortality and the abortion in the descendants.
\end{abstract}

Keywords: Consanguinity, morbidity, mortality, fertility, Tiflet

Résumé

Bien que les résultats de plusieurs recherches confirment l'implication de la consanguinité dans les problèmes de santé, certains pays conservent encore ce comportement matrimonial. La présente étude a pour objectif l'analyse de l'impact de la consanguinité sur la santé des descendants dans la ville de Tiflet et ses régions (Nord-ouest du Maroc).

Une enquête prospective a été réalisée sur 1000 familles échantillonnées au hasard entre juin et novembre de l'année 2012. Le calcul du taux de la 
mortalité a concerné 3345 descendants nés vivants, alors que l’étude des effets biologiques de la consanguinité a été faite sur un total de 3241 descendants. Les résultats de cette étude révèlent une association significative entre la consanguinité et l'incidence des affections de santé, à savoir la morbidité, la mortalité et l'avortement chez les descendants.

Mots clés : Consanguinité, morbidité, mortalité, fécondité, Tiflet

\section{Introduction}

Deux individus A et B sont dit apparentés s'ils ont au moins un ancêtre commun. On appelle mariage consanguin, l'union entre deux individus apparentés. Les enfants qui naissent de tels mariages sont appelés consanguins et sont plus souvent homozygotes que ne le voudrait le hasard. Il est connu depuis longtemps que la consanguinité augmente le degré d'homozygotie et favorise l'apparition de gènes délétères dans le pool génétique de la population (Biemont, 1979 ; Klat, 1986 ; Solignac et al., 1995 ; Bener et al., 2001).

Les effets de la consanguinité sur la mortalité infantile retiennent l'attention des médecins, biologistes et généticiens depuis longtemps. L'American Medical Association réalisa la première enquête sur le sujet en 1856. Elle demande à ses membres de colliger des données de mortalité et de morbidité parmi des familles consanguines et des familles témoins. Les données sont publiées par Bemiss en 1858, mais il faut attendre un siècle avant que Sutter et Tabah n'en fassent une analyse détaillée en 1951 (Sutter et Tabah, 1951).

Depuis longtemps, les généticiens se sont intéressés aux conséquences des mariages consanguins notamment en ce qui a trait à la fécondité, la mortalité et la morbidité. Ceci a entraîné une abondance de publications depuis la fin du XIXème siècle (Khoury et al., 1987; Bittles et al., 1991; Khlat et Khoury, 1991). En effet, les études réalisées pour estimer les effets biologiques de la consanguinité s'accordent sur le fait que les effets délétères de la santé liés à la consanguinité sont causés par l'expression de gènes récessifs rares, hérités d'un ou plusieurs ancêtres communs. Par conséquent, dans les populations où la consanguinité est largement répandue, l'augmentation du taux de morbidité et de mortalité imputable à l'action de gènes délétères.

L’objectif principal de la présente étude est de prendre le relai des autres études réalisées au Maroc sur ce sujet et de déterminer les effets biologiques de la consanguinité sur les descendants dans la population de Tiflet (Nord-ouest du Maroc). 


\section{Matériels et méthodes}

Une enquête prospective a été menée sur 1000 couples échantillonnés aléatoirement entre juin et novembre de l’année 2012 dans la ville de Tiflet. L’enquête a été réalisée à l'aide d'un questionnaire établi par le laboratoire de Génétique et Biométrie de la Faculté des Sciences de Kénitra. Dans cette étude, le calcul du taux de la mortalité a concerné 3345 descendants nés vivants, alors que l'étude des effets biologiques de la consanguinité a été faite sur un total de 3241 descendants. En effet, nous avons recensé toutes les maladies susceptibles d'être en relation avec des facteurs génétiques chez 1393 descendants issus de mariages consanguins et chez 1848 descendants témoins issus de mariage entre non apparentés.

Les différentes catégories de mortalité faisant l'objet de la présente étude sont :

- $\quad$ La mortalité infantile = (Nombre de décès avant un an / Nombre de naissances vivantes)*1000.

- $\quad$ La mortalité juvénile = (Nombre de décès entre 1 et 5 ans / Nombre de naissances vivantes)*1000

La mortalité chez les individus ayant l'âge supérieur à 5 ans = (Nombre de décès a un âge supérieur à 5ans / Nombre de naissances vivantes)*1000.

Pour faire le suivi de la variation de la proportion d'homozygotie selon l'état des descendants, nous avons fait appel au coefficient de consanguinité au sein de chaque catégorie de malades. Le coefficient de consanguinité d'un individu (I) se mesure à partir de la probabilité pour que les deux gènes qu'il possède, à un locus donné, soient identiques par descendance (Malécot 1948 ; jacquard 1968) :

$$
\mathrm{F}_{\mathrm{I}}=\Sigma(1 / 2)^{\mathrm{np}+\mathrm{nm}+1} \times\left[1+\mathrm{F}_{\mathrm{A}}\right] \text { où }
$$

$\mathrm{n}_{\mathrm{p}}$ désigne le nombre de générations qui séparent le père de l'individu i de l'ancêtre $A$;

$\mathrm{n}_{\mathrm{m}}$ désigne le nombre de générations qui séparent la mère de l’individu i de l'ancêtre A ;

$\mathrm{F}_{\mathrm{A}}$ désigne le coefficient de consanguinité de l’ancêtre A.

\section{Résultats et discussion}

L'association entre la consanguinité et l'état de santé de la descendance a été soulignée par plusieurs auteurs (Freire-Maia, 1964 ; Bonaïti et al., 1978 ; Khoury et al., 1989 ; Freire-Maia, 1990 ; Agarwal et al., 1991 ; Jain et al., 1993 ; Teebi, 1994 ; Vogel et Motulsky, 1997 ). Les études sur l'effet de la consanguinité ont porté principalement sur l’impact sur la mortalité, la mortalité infanto-juvénile, la reproduction et les désordres mendéliens (Mi et al., 1965 ; Edmond et De Braekeleer, 1993 ; Charlesworth 
et Hughes, 1999 ; Charlesworth et Charlesworth, 1999 ; Hussain et al., 2001).

\section{Étude de l’impact de la consanguinité sur la morbidité. Étude à l'échelle de la population totale.}

Dans notre échantillon de 1000 couples, nous avons enregistré une fréquence de 38,9\% des unions consanguines avec un coefficient de consanguinité de 5645,50.10 (Abbad, Z., et al., 2016).

Pour déterminer l'impact de la consanguinité sur la morbidité dans la descendance totale constituée des enfants issus de mariages consanguins et des mariages non consanguins, nous avons subdivisé les différentes atteintes morbides en trois catégories, à savoir les désordres congénitaux, le retard mental et les maladies multifactorielles.

La figure 2 montre que la morbidité globale atteint $6.20 \%$ et que les maladies multifactorielles représentent presque la moitié des atteintes morbides avec un taux de 3.18\%. Par contre, le retard mental et les désordres congénitaux ne représentent que $1,7 \%$ et $1,64 \%$ respectivement.

Dans une étude menée sur quatre populations marocaines, dans la région de Doukkala, Talbi, J., (2007) a déclaré un taux de $17.66 \%$ de morbidité globale et des taux plus élevés que ceux trouvés dans notre étude dans les deux catégories "désordres congénitaux » $(4.55 \%)$ et "maladies multifactorielles » (15.21\%).

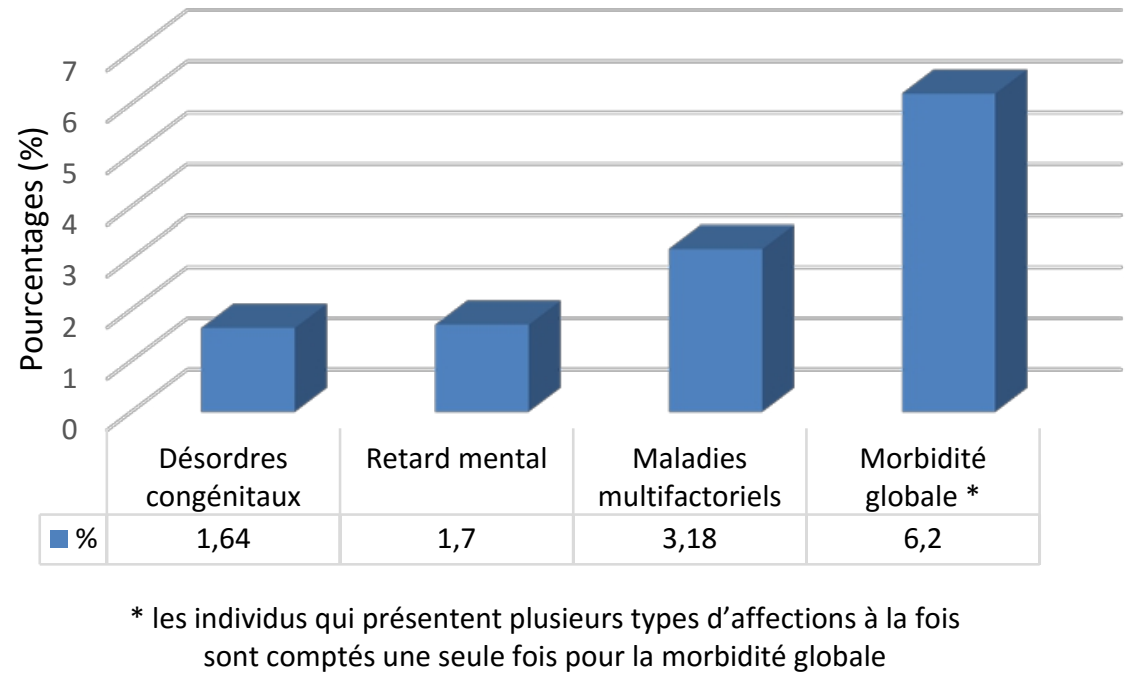

La figure 2 : Prévalence de la morbidité de chaque catégorie de maladies chez la descendance totale. 


\section{Étude sur la base du coefficient de consanguinité}

L'importance des modifications génotypiques dépend de la valeur du coefficient de consanguinité $\mathrm{F}$, donc de la structure des mariages consanguins. Plus les partenaires d'un mariage sont apparentés, plus la probabilité qu'un enfant issu de ce mariage possède deux copies identiques par descendance héritées d'un ancêtre commun à ses père et mère est grande. C'est le coefficient de consanguinité (Malécot, 1966; Jacquard, 1970).

Comme le montrent les résultats du tableau 1 , nous constatons que le coefficient de consanguinité des enfants malades est supérieur à celui de la totalité des descendants consanguins. Ce résultat concorde avec celui trouvé par Talbi, J., (2007).

Les malades affectés par des atteintes multifactorielles et ceux souffrant des désordres congénitaux ont des coefficients de consanguinité presque similaires $\left(5937,50.10^{-5}\right.$ et $5959,34.10^{-5}$ respectivement), ces coefficients sont plus élevés par rapport à celui des descendants atteints de retard mental $\left(4843,75.10^{-5}\right)$.

Par ailleurs, nous constatons que, chez les deux sexes, le coefficient de consanguinité chez les descendants malades est supérieur à celui chez les descendants indemnes (figure 3). Le test statistique Khi2 indique que la consanguinité n'a pas d'effet significatif sur le sexe (Khi2=2,000; $>0,05$ ).

Nos résultats ne s'accordent pas avec ceux cités par Talbi, J., (2007) qui a déclaré que les individus atteints de désordres congénitaux présentent un coefficient plus élevé que celui des individus atteints de maladies à caractère multifactoriel, alors que les descendants atteints de retard mental présentent le coefficient le plus élevé dans la région de Doukkala. Le même auteur a confirmé que l'effet de la consanguinité chez le sexe féminin est plus accentué par rapport à celui chez le sexe masculin.

\begin{tabular}{|c|c|c|c|c|c|c|}
\hline & \multirow[b]{2}{*}{ Total } & \multicolumn{3}{|c|}{ Consanguins } & \multicolumn{2}{|c|}{ Non consanguins } \\
\hline & & $\mathrm{n}$ & $\%$ & $\mathrm{~F}$ & $\mathrm{n}$ & $\%$ \\
\hline Unions & 1000 & 389 & 38.90 & $5645.50 .10^{-5}$ & 611 & 61.1 \\
\hline Descendances & 3241 & 1393 & 42.98 & $5732.20 .10^{-5}$ & 1848 & 57.01 \\
\hline Malades & 201 & 128 & 63.68 & $6029.41 .10^{-5}$ & 73 & 36.32 \\
\hline *Atteintes multifactorielles & 103 & 45 & 43.69 & $5937.50 .10^{-5}$ & 58 & 56.31 \\
\hline$*$ Retard mental & 55 & 50 & 90.91 & $4843.75 .10^{-5}$ & 5 & 9.09 \\
\hline *Désordre congénital & 53 & 43 & 81.13 & $5959.34 .10^{-5}$ & 10 & 18.87 \\
\hline Malades de sexe féminin & 84 & 51 & 60.71 & $5974.40 .10^{-5}$ & 33 & 39.29 \\
\hline Malades de sexe masculin & 117 & 77 & 65.81 & $5845.00 .10^{-5}$ & 40 & 34.19 \\
\hline & & & & par catégorie & & \\
\hline
\end{tabular}

Tableau 1 : Pourcentages et coefficients de consanguinité dans les différentes catégories de maladies chez les descendants. 


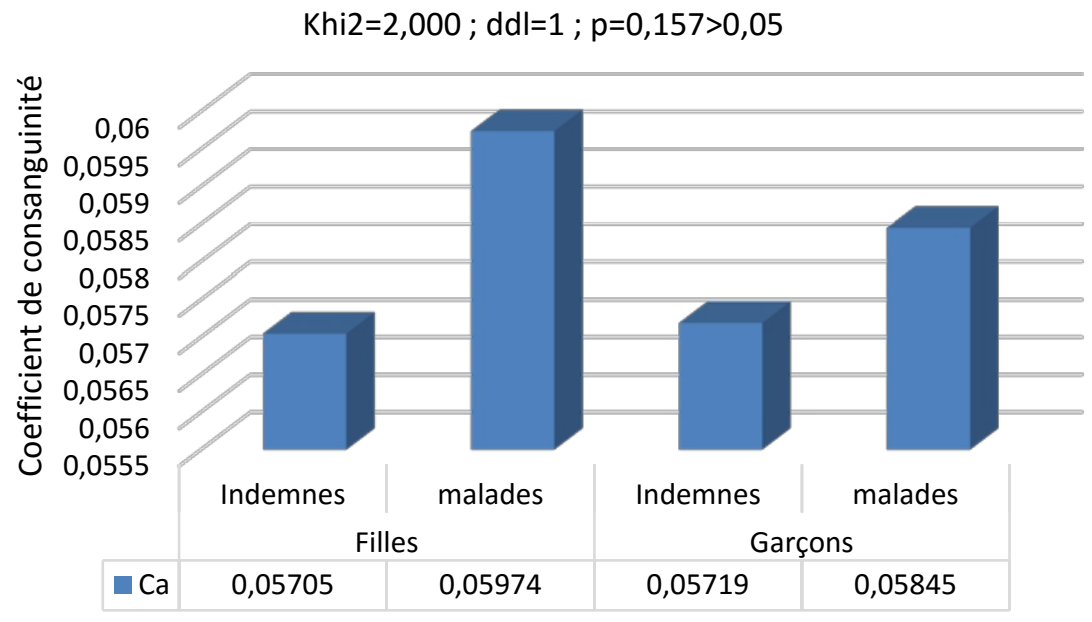

Ca: Coefficient de consanguinité

Figure 3 : Coefficient de consanguinité en fonction du sexe et de l'état de santé des descendants.

L'analyse des résultats consignés dans le tableau 2 révèle une relation significative entre la consanguinité et certaines maladies $(p<0,05)$. En effet, les malades consanguins atteints du désordre congénital (3.10\%) et de retard mental (3.59\%) représentent des pourcentages plus élevés par rapport à ceux des malades non consanguins qui ne dépassent pas $0.50 \%$. Les résultats du test statistique Khi2 montrent qu'il y a une association entre la consanguinité et les atteintes morbides des catégories « retard mental » (Khi2=32,001; $\mathrm{p}=0.000)$ et " désordre congénital » $(\mathrm{Khi} 2=52,443$; $\mathrm{p}=0.000)$. De même, il est à noter que la consanguinité a un effet significatif sur la morbidité globale chez les descendants consanguins (Khi2=73,471; $\mathrm{p}=0.000$ ).

Tableau 2: Prévalence des affections morbides chez la descendance consanguine et non consanguine.

\begin{tabular}{|c|c|c|c|c|c|c|c|}
\cline { 2 - 6 } \multicolumn{1}{c|}{} & \multicolumn{2}{c|}{ Consanguins } & \multicolumn{2}{c|}{ Non consanguins } & \multirow{2}{*}{ ddl } & \multirow{2}{*}{ Chi2 } & \multirow{2}{*}{ P } \\
\cline { 2 - 8 } \multicolumn{1}{c|}{} & $\mathrm{n}$ & $\%$ & $\mathrm{n}$ & $\%$ & 1 & 32.001 & 0.000 \\
\hline $\begin{array}{c}\text { Désordre } \\
\text { congénital }\end{array}$ & 43 & 3.10 & 10 & 0.50 & 1 & & \\
\hline Retard mental & 50 & 3.59 & 5 & 0.27 & 1 & 52.443 & 0.000 \\
\hline $\begin{array}{c}\text { Maladies } \\
\text { multifactorielles }\end{array}$ & 45 & 3.23 & 58 & 3.14 & 1 & 0.022 & 0.480 \\
\hline Morbidité globale & 128 & 9.19 & 73 & 3.95 & 1 & 37.471 & 0.000 \\
\hline
\end{tabular}

Nos résultats corroborent ceux de Heston (1970) qui avait déclaré la consanguinité comme facteur de risque pour les maladies mentales (Emery, 1986). De même, Semanova, (1971) avait rapporté que 39,8\% d'enfants consanguins souffrent de troubles mentaux, de surdité et de retards mentaux 
sévères comparés à 5,3\% seulement d'enfants non consanguins (Van Der Berghe, 1983).

Sidi-Yakhelf et al. en 2012 a cité que le cancer et les troubles mentaux sont associés significativement à la consanguinité ainsi que le diabète insulinodépendant et les malformations congénitales qui est à la limite de la signification avec une P-value de 0,054 et 0,051 respectivement.

Quant aux maladies multifactorielles, les résultats de la présente étude (tableau 2) révèlent que les pourcentages de ce type d'atteinte sont similaires chez les descendants apparentés et les non apparentés (3.23\% et 3.14\% respectivement). En effet, L'analyse statistique reposant sur le Khi2 effectué sur nos données ne montre aucune différence significative entre les proportions des maladies multifactorielles chez les enfants apparentés et les non apparentés (Khi2=0,022, $\mathrm{p}=0.480$ ). Ces résultats pourraient être dus aussi à l'effet de l'environnement et/ou à la structure génétique de notre population d'étude.

Dans la région de Doukkala ( Maroc), Talbi (2007) a conclu une augmentation importante de la fréquence des maladies à caractère multifactoriel chez les consanguins. Ces résultats concordent également avec ceux publiés par Rudan et al., (2003a).

D’un autre côté, nous avons essayé d'estimer le degré d'intervention de la consanguinité dans l'apparition de certaines maladies très répandues dans notre population.

A la lumière des résultats de la figure 4, nous constatons que les pourcentages de certaines atteintes de la catégorie « désordres congénitaux » sont élevés chez les descendants consanguins par rapport à ceux des enfants non consanguins, c’est le cas de la paraplégie cérébrale et du strabisme. Toutefois, certaines maladies sont présentes chez les descendants consanguins et parfaitement absentes chez les non consanguins, c'est le cas de la phénylcétonurie, la surdi-mutité, hydrocéphalie et la fissure labiale.

En outre, les fréquences de certaines maladies des atteintes de la catégorie «maladies multifactorielles » telles que l'autisme, l'épilepsie, le glaucome et la lèpre sont élevées chez les descendants consanguins en comparaison avec celles des descendants non consanguins. Toutefois, la tuberculose et le diabète sont accentués chez les descendants non consanguins.

A l'opposé de nos résultats concernant le diabète, certains auteurs tels que Moussouni, A. (2011) ; Mortad N. et al., (2013) ; Benallègue et Kedji (1984) et Bittles (2001) ont déclaré que les descendants consanguins semblent être plus prédisposés au diabète.

En Tunisie, l’étude de Mongalgie et ses collaborateurs (1991) sur le diabète infantile démontre que $48 \%$ des enfants souffrent de cette maladie en raison de la consanguinité. 


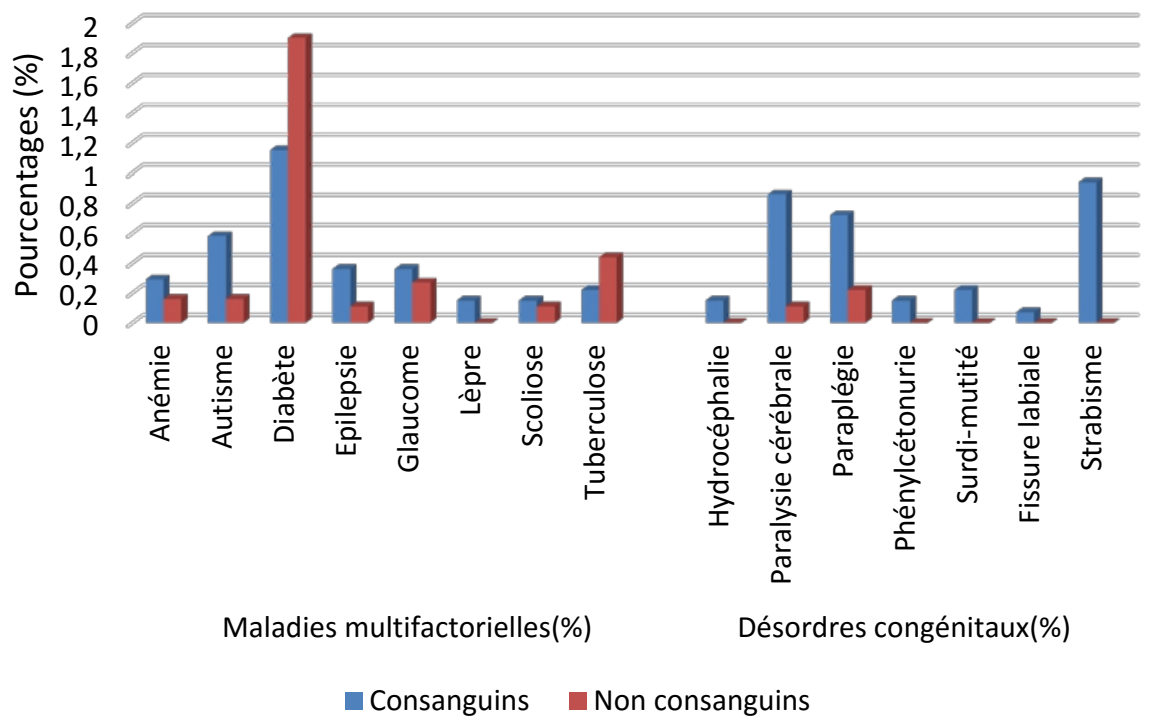

Figure 4: Prévalence de chaque type de maladie chez la descendance consanguine et non consanguine.

D'ailleurs, Moussouni, A., (2011), a signalé une relation hautement significative entre la consanguinité et certaines maladies telles que le diabète, l'hypertension artérielle, l'asthme, les cardiopathies, l'allergie et les maladies congénitales. Talbi, J., (2007),de sa part, a démontré que, parmi les maladies multifactorielles, les risques d'atteinte d'asthénie, d'hypertension artérielle, d'allergie, de problèmes génitaux et de problèmes d'audition chez les consanguins, sont les plus élevés.

\section{Étude de l’impact de la consanguinité sur la mortalité.}

L'existence d'une association entre la consanguinité et de multiples anomalies létales devrait en toute logique conduire à une mortalité globale plus élevée des enfants issus de mariages consanguins. Toutefois les conséquences d'ordre démographique sont difficiles à tester du fait d'un rôle important des facteurs environnementaux, au sens large, dans le déterminisme de la mortalité (Moussouni, A., 2011).

\section{Etude à l'échelle de la population totale.}

Les résultats portés dans la figure 5 révèlent un taux de mortalité élevé chez les enfants issus des couples consanguins (21.52\%o) par rapport aux enfants issus des couples non consanguins (9.57\%o). En outre, le taux de mortalité chez les couples consanguins du premier degré est nettement important que celui enregistré chez les couples consanguins du deuxième degré. Ces résultats controversent ceux de Moussouni, A., (2011), qui a 
montré que le taux de mortalité chez les couples consanguins du second degré apparaît clairement important que celui enregistré chez les couples consanguins du premier degré dans la population de Sabra dans l'Ouest algérien.

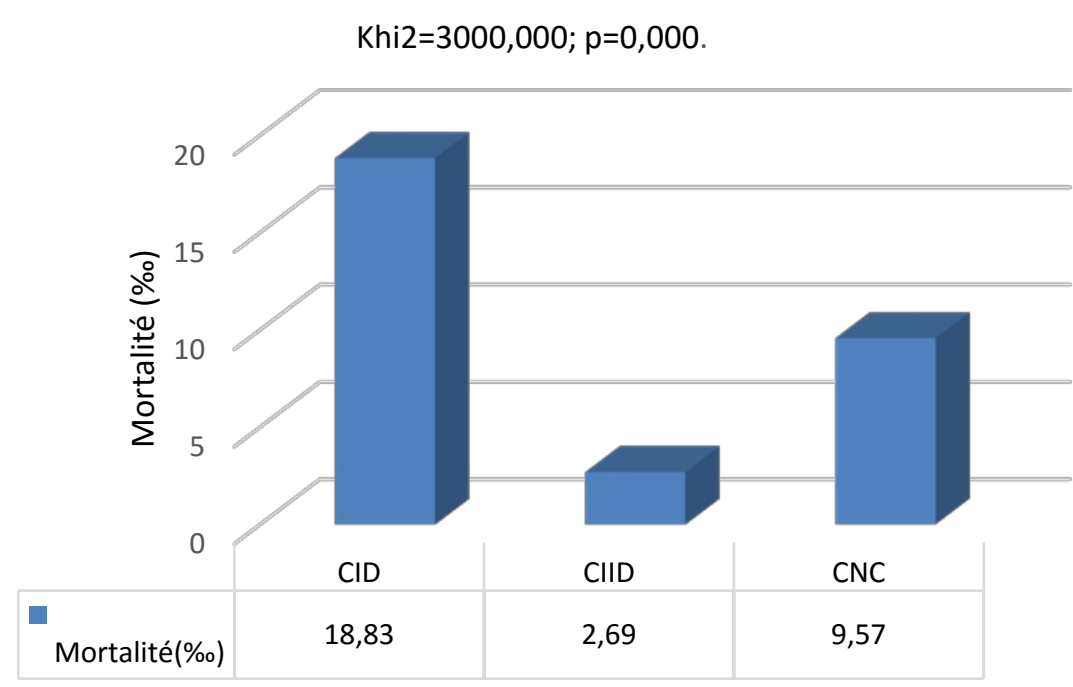

CID: Descendants issus des cousins germains

CIID: Descendants issus des cousins issus de germains et des cousins inégaux

CNC: Descendants issus des maraiges non consanguins

Figure 5 : Répartition de la mortalité des descendants issus de familles consanguines et non Consanguines.

Le test statistique Khi2 témoigne une relation significative entre la consanguinité et la mortalité chez les descendants dans la population de Tiflet (Khi2 $=3000.000 ; \mathrm{p}=0.000$ ). Nos résultats sont en harmonie avec ceux cités par Mortad N. et al., (2013) qui a bien montré que plus les couples sont consanguins, plus ils sont touchés par la mortalité dans la population de Msirda dans l'extrême Ouest algérien. Dans le même sens, Talbi, J., (2007) a conclu que la moyenne des décès chez la descendance consanguine est significativement plus élevée par rapport à la descendance non consanguine. Cependant, De Braekeleer., (2005) a conclu que la surmortalité néonatale précoce et infantile n'est pas déterminante parmi la descendance des mariages consanguins ce qui ne corrobore pas nos résultats.

\section{Etude sur la base du coefficient de consanguinité.}

D’après les résultats du tableau 3 , nous observons que le coefficient de consanguinité des individus décédés est supérieur à celui des individus vivants, ce qui reflète que la proportion de l'homozygotie est élevée chez les 
descendants décédés. De même, la mortalité des enfants de " 5 ans et plus » présente un coefficient de consanguinité supérieur à ceux enregistrés dans les catégories des décès infantiles et juvéniles. Par ailleurs les coefficients de ces deux dernières catégories sont presque similaires au coefficient de consanguinités des descendants vivants.

\begin{tabular}{|c|c|c|c|c|c|c|}
\cline { 3 - 7 } \multicolumn{1}{c|}{} & \multirow{2}{*}{ Total } & \multicolumn{3}{c|}{ Consanguins } & \multicolumn{2}{c|}{ Non consanguins } \\
\cline { 3 - 7 } \multicolumn{1}{c|}{} & & $\mathrm{n}$ & $\%$ & $\mathrm{~F}$ & $\mathrm{n}$ & $\%$ \\
\hline Vivants & 3241 & 1393 & 42.98 & $6064,30.10^{-5}$ & 1848 & 57.01 \\
\hline Décédés & 104 & 72 & 69.23 & $6119,78.10^{-5}$ & 32 & 30.77 \\
\hline $0-12$ mois & 67 & 47 & 70.15 & $6083,77.10^{-5}$ & 20 & 29.85 \\
\hline 1 1-5ans & 27 & 17 & 62.96 & $6066,20.10^{-5}$ & 10 & 37.04 \\
\hline$>$ 5ans & 10 & 8 & 80 & $6250,00.10^{-5}$ & 2 & 20 \\
\hline \multicolumn{7}{c}{$\mathrm{F}:$ coefficient de consanguinité ; $\mathrm{n}:$ Effectif } \\
\hline
\end{tabular}

Tableau 3 : Pourcentages et coefficients de consanguinité chez les différentes catégories d’âge de décès chez les decsendants.

Talbi J. et al., (2007) a rapporté que, dans son estimation du coefficient de consanguinité pour chaque catégorie de mortalité, la proportion d'homozygotie chez les individus décédés est nettement plus élevée par rapport à celle des individus vivants. Cette proportion reste plus importante dans les cas de mortalité infantile et de mortalité juvénile. Il a indiqué aussi que les individus décédés après l'âge de cinq ans présentent un coefficient de consanguinité inférieur à celui des individus vivants.

Les résultats recueillis dans le tableau 4 montrent également que les taux de mortalité globale, de mortalité infantile, de mortalité juvénile et même de la mortalité des enfants de 5 ans et plus, sont plus élevés chez les consanguins par rapport aux non consanguins $(p<0,005)$. Nos résultats concordent avec ceux de plusieurs études menées dans les pays d'Asie, qui ont montré les effets de la consanguinité sur la mortalité infantile en Arabie Saoudite ( Wong, Anokute 1990), en Turquie (Guz et al. 1989 ;Tuncbilek, Koc 1994), au Pakistan (Shami et al. 1989 ; Grant, Bittles 1997 ; Hussain et al. 2001) et en Inde ( Verma et al. 1992 ; Banerjee, Roy 2002).

\begin{tabular}{|c|c|c|c|c|c|c|c|}
\cline { 2 - 6 } \multicolumn{1}{c|}{} & \multicolumn{2}{c|}{ Consanguins } & \multicolumn{2}{c|}{ Non Consanguins } & \multirow{2}{*}{ ddl } & \multirow{2}{*}{ Chi2 } & \multirow{2}{*}{ p } \\
\cline { 2 - 8 } \multicolumn{1}{c|}{} & $\mathrm{n}$ & \%o & $\mathrm{n}$ & \%o & & & \\
\hline Décédés & 72 & 49 & 32 & 17 & 1 & 28.208 & 0.000 \\
\hline 0 -12mois & 47 & 32 & 20 & 11 & 1 & 19.289 & 0.000 \\
\hline 1-5ans & 17 & 12 & 10 & 5 & 1 & 4.062 & 0.035 \\
\hline$>5$ & 8 & 5 & 2 & 1 & 1 & 5.341 & 0.023 \\
\hline
\end{tabular}

Tableau 4 : Taux de mortalité chez les descendants consanguins et non consanguins.

Contrairement à ce qui précède, Al- Awadi et al., (1986) et d'autres auteurs ont conclu, à l'issue d'études sur la mortalité prénatale et postnatale 
au Sud de l'Inde et au Koweït, une indépendance entre la consanguinité et la mortalité (Yaqoob et al., 1998).

Au nord de l'Afrique, Hami, H. et al., (2007) a conclu également que l'écart entre les deux groupes (consanguins et non consanguins) reste non significatif dans les deux régions marocaines, «Rabat-Salé-ZemmourZaer »et « Gharb-Chrarda-Beni-Hssen », bien que les couples consanguins présentent des taux plus élevés de mortinatalité. De même, Sidi-Yakhlef, A., et al., (2012) a montré une mortinatalité plus élevée chez les enfants issus des couples non consanguins par rapport aux consanguins dans la population de Oulhaça dans l’Ouest algérien.

Les auteurs attribuent généralement cette absence d'effet aux mécanismes d'adaptation à la consanguinité par élimination progressive des gènes létaux au fur et à mesure des générations consanguines (Khlat, 1986 ; Bener et al., 2001 ; Bittles et al., 2001 ; Rittler et al., 2001).

Par ailleurs, la plus part des auteurs qui ont examiné la mortalité prénatale et post-natale s’accordent sur le fait que la consanguinité augmente inéluctablement le taux de mortalité (Hussain, 1998 ; Yaqoob et al., 1998).

\section{Étude de l'impact de la consanguinité sur l’avortement.}

Un avortement spontané est l'expulsion non provoqué du produit de conception avant la date de la viabilité foetale. C'est ce qu'on appelle une fausse couche. Du point de vue de l'âge gestationnel, la date de viabilité foetale a été le sujet de beaucoup de débats scientifiques et légaux, si bien que cette définition varie avec les législations et les habitudes médicales. Cependant, on considère qu'un foetus est potentiellement viable s'il pèse plus de $500 \mathrm{~g}$ et/ou est âgé de plus de 20 semaines d'aménorrhée (définition OMS).

A travers notre enquête, nous avons compté le nombre des avortements sans prendre en considération le nombre de semaines des fausses couches vu que la plupart des femmes ne se rappellent plus la date exact de l'évènement.

Les résultats mentionnés dans la figure 6 révèlent un taux d'avortement élevé chez les couples consanguins (57.7\%) par rapport à celui des couples non consanguins (36.8\%). De plus, si nous prenons en considération le degré de répétabilité, le pourcentage d'avortement est plus important chez les couples consanguins du premier degré que chez ceux du second degré. Ces résultats mettent en évidence un effet significativement néfaste de la consanguinité sur l'avortement au sein de la population de Tiflet ( $<<0,05)$. Nos résultats corroborent ceux cités par Mortad, N. et al., (2013) qui a trouvé que plus les couples sont consanguins, plus ils sont touchés par l'avortement. Autrement dit, la consanguinité exerce un effet plus ou moins 
significatif sur la possibilité que les couples soient touchés par ce phénomène d'avortement.

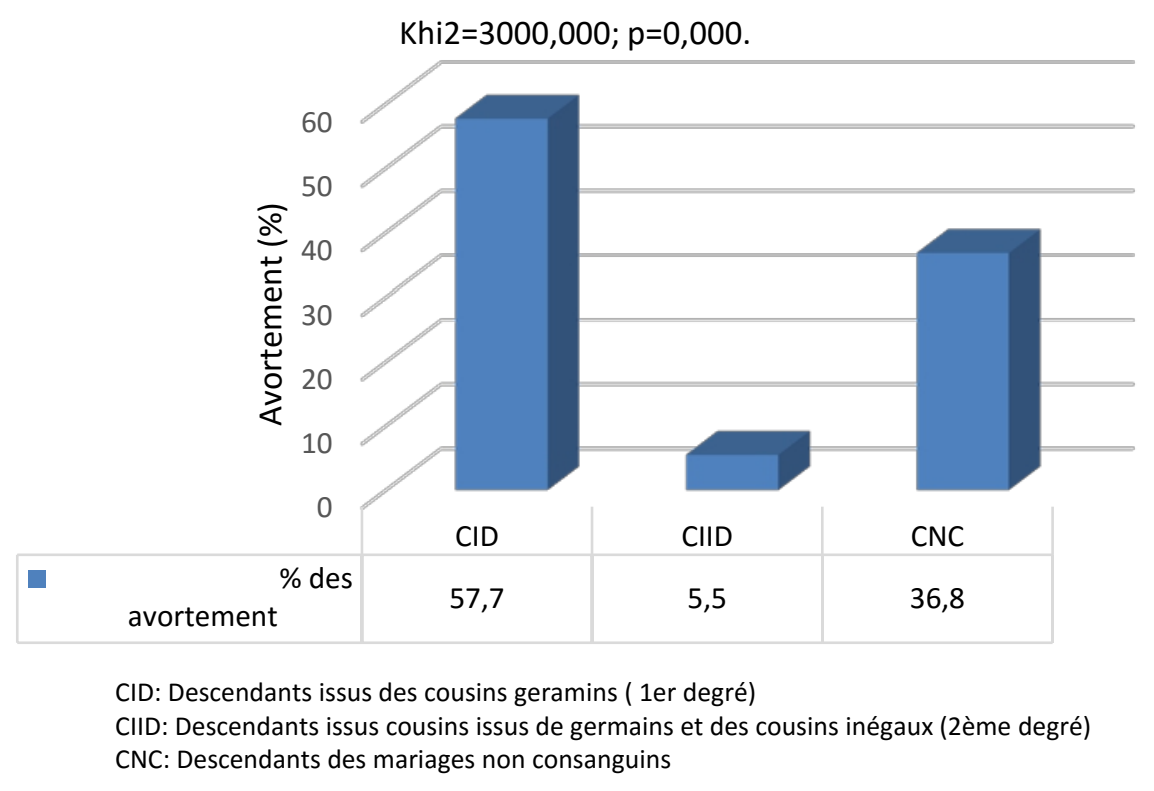

Figure 6. : Taux d’avortement et lien de parenté des conjoints.

Hussain, (1998) et Bener et al., (2001) ont déclaré que le taux des avortements spontanés est d'autant plus élevé dans les familles où la consanguinité se perpétue depuis plusieurs générations à cause de l'effet d’homozygotie croissante sur le développement du fœtus. De même, Une étude réalisée en Turquie révèle que la proportion d'avortements est deux fois supérieure chez les couples consanguins du premier degré par rapport aux non consanguins (Hussain, 1998).

Des résultats similaires ont été obtenus à l'issue d'études effectuées en Irak (Hussain, 1998) et au sud de l'Inde (Zain et al., 1988). Cependant, une autre étude menée sur la Jordanie révèle que le taux d'avortement n'a pas été affecté par la consanguinité (Khoury et Massad, 2000; Ghazi et al., 2009). D'autres recherches ont rapporté des résultats similaires (Al Awadi et al., 1986; Hamamy et al., 1986; Khlat, 1988 ; Abdulrazzaq et al, 1997; Saad et Jauniaux, 2002 ; Kerkeni et al., 2007; Ghazi et al., 2009).

\section{Conclusion}

l'enquête que nous avons menée pour évaluer l'impact biologique des mariages consanguins, dans la population de Tiflet où le taux de consanguinité atteint 38,9\%, met en exergue, d'un côté, un effet néfaste de la consanguinité sur l'avortement, sur la mortalité infantile, sur la mortalité juvénile et sur la mortalité des descendants de 5 ans et plus et, d'un autre 
côté, une relation hautement significative entre la consanguinité et quelques maladies, à savoir le retard mental, la paraplégie cérébrale, le strabisme, la phénylcétonurie, la surdi-mutité et l’hydrocéphalie.

Par ailleurs, nos résultats ont montré que le taux de mortalité chez les couples consanguins du premier degré apparaît clairement important que celui enregistré chez les apparentés du second degré et, inversement, le taux d'avortement chez les couples consanguins du second degré est supérieur à celui enregistré chez les apparentés du premier degré.

A travers cette étude, nous avons conclu, également, que la consanguinité n'a pas d'effet sur le sexe dans notre échantillon.

En dépit de ces résultats qui témoignent, du point de vue génétique, l'impact de la consanguinité sur la santé des descendants, d’autres facteurs d’ordre socio-économique, culturel, environnemental et religieux pourraient intervenir dans ce phénomène dans la population de Tiflet.

\section{References:}

Abbad Z., Drissi A., Soulaymani A., Khadmaoui A., Oukarroum A., (2016) «Études Des Caractéristiques Et Des Déterminants Des Mariages Consanguins Dans La Ville De Tiflet (Maroc)». European Scientific Journal February 2016 edition vol.12, No.9 ISSN: 1857 - 7881 (Print) e - ISSN 1857- 7431.

Abdulrazzaq Y.M, Bener A, al-Gazali L.I, al-Khayat A.I, Micallef R, Gaber T., 1997. A study of possible deleterious effects of consanguinity. Clin Genet, 51:167-173.

Agarwal S.S., Singh U., Singh P.S., Singh S.S., Das V., Sharma A., Mehra P., Chandravati G.K., Malik P. et Misra P.K., 1991: Prevalence and spectrum of congenital malformations in a prospective study at a teaching hospital. Indian Journal of Medical Research, 94, 413-419.

Al-Awadi S.A, Naguib K.K, Moussa M.A, Farag T.I, Teebi A.S, el-Khalifa M.Y., 1986. The effect of consanguineous marriages on reproductive wastage. Clin Genet, 29: 384-388.

Benerjee S.K., Parentel consanguinity and offspring mortality: the search for possible linkage in the Indian context. Asia-pacific population journal 2002, 17(1), 17-38.

Bener A, Denic S, AL-MazSouei M., 2001. Consanguinity and family history of cancer in children with Leukemia and lymphomas. American cancer society, 92: 1-6.

Benallegue A, Kedji F, 1984. Consanguinity and public health. Algerian Study. Arch Fr Pediatr, 41: 435-440.

Biemont C, 1979. Effets et mécanismes de la consanguinité chez Drosophila Melanogaster. Thèse de doctorat -ès-sciences. Univ. Lyon. 
Bittles AH., 1991: Consanguinity: A major variable in studies of North African reproductive behaviour, morbidity and mortality? In: Proceedings of the Demographic and Health Surveys World Conference, Columbia, MD: IRD/Macro International, 1, 321-341.

Bittles, A. (2002). Endogamy, Consanguinity and community genetics. 81 (3), 91-98.

Bonaïti C., Demenais F., Briard M.L. et Feingold J., 1978: Consanguinity in multifactorial inheritance. Application to data on congenital glaucome. Human Heredity, 28, 361-71.

Charlesworth B. et Charlesworth D., 1999: The genetic basis of inbreeding depression. Genetic Research, 74, 329-40.

Charlesworth B. et Hughes K.A., 1999: The maintenance of genetic variation in life history traits. In: Singh R.S., Krimbas C.B., eds. Evolutionary genetics: from molecules to morphology, vol 1. Cambridge: Cambridge University Press.

Edmond M. et De Braekeleer M., 1993: Inbreeding effects on prereproductive mortality: A case-control study in Saguenay-Lac-Saint-Jean (Quebec, Canada) based on a population registry 1838-1971. Annals Human Biology, 20, 535-543.

Emery A.E.H, 1986. Abrégés génétique médicale. Masson, Pages 131-154.

Freire-Maia N. et Freire-Maia A., 1964: Estimate of the load of mutations disclosed by inbreeding. Genetics, 50, 527-9.

Freire-Maia N., 1990: Five landmarks in inbreeding studies. American Journal of Medical Genetics, 35, 118-20.

Ghazi O.T, Pratibha N, Tasneem O, Mahmoud T.A.A, Najib A.K and Hanan A.H., 2009. Consanguinity and reproductive health among Arabs. Reproductive Health, 6: 17 doi:10.1186/1742-4755-6-17.

Jacquard, A (1968). Panmixie et consanguinité. Quelques précisions de langage. Population, 6, 1065-1090.

Jacquard A., 1970: Structure génétique des populations. Masson, Paris, pp.399.

Jain V.K., Nalini P., Chandra R. et Srinivasan S., 1993: Congenital malformations, reproductive wastage and consanguineous mating. Austral New Zeal J Obstet Gynaecol, 33, 33-36.

Grant J.C., Bittles A.H., The comparative role of consanguinity in infant and childhood mortality in Pakistan. Annals of Human genetic 1997, 61(2), 143149.

Guz K. Dedeoglu N. LuleciG., The frequency and medical effects consanguineous marriages in Antalya, Turkey. Hereditas 1989,111(1), 7983; 
Hamamy H, Al-Bayati N, Al-Kubaisy W, 1986. Consanguineous marriages in Iraqi urban population and the effect on pregnancy outcome and infant mortality. Iraqi Med J, 34: 76-80.

Hami, H. et al., (2007). Déterminants des mariages consanguins au Maroc, cas de deux régions du Nord-ouest «Région de Rabat-Salé-Zemmour-Zaer et Région de Gharb-Chrarda-Beni-Hssen ». Thèse pour l'obtention du diplôme de doctorat. P 103.

Hussain, R. (1998). The role of consanguinity and inbreeding as a determinant of spontaneous abortion in Karachi, Pakistan. Annals of Human Genetics , 62, 147-157.

Hussain R., Bittles A.H. et Sullivan S., 2001: Consanguinity and early mortality in Muslim populations of India and Pakistan. American Journal of Human Biology, 13, 777-787.

Kerkeni E, Monastiri K, Saket B, Guediche M.N, Ben C.H., 2007. Interplay of socio-economic factors, consanguinity, fertility, and offspring mortality in Monastir, Tunisia. Croat Med J,48: 701-707.

Khlat, M. (1986). Les mariages consanguins à Beyrouth : Structure et conséquences biologiques. Univ de Lyon :Thèse de doctorat .

Khlat M, 1988. Consanguineous marriage and reproduction in Beirut, Lebanon. American Journal of Human Genetics, 43 (2) : 188-196.

Khlat M. et Khoury M., 1991: Inbreeding and diseases: Demographic, Genetic and Epidemiologic Perspectives. Epidemiologic Reviews, 28-41.

Khoury M.J., Cohen B.H., Diamond E.L., Chase G. et McKusick V., 1987: Inbreeding and prereproductive mortality in the Old Order Amish. I. Genealogic epidemiology of inbreeding. American Journal of Epidemiology, 125, 453-61.

Khoury M.J., Flanders W.D., Greenland S. et Adams M.J., 1989: On the measurement of susceptibility in the epidemiologic studies. American Journal of Epidemiology, 129, 183-90.

Khoury S.A, Massad D.F, 2000. Consanguinity, fertility, reproductive wastage, infant mortality and congenital malformations in Jordan. Saudi Med J,21:150-154.

Malécot G., 1948: Les mathématiques de l’hérédité. ed. Masson, Paris. Malécot G., Probabilité et Hérédité, 1966. Presses universitaires de France.

Mi M.P., Azevêdo E., Krieger H. et Morton N.E., 1965: Malformations in north-eastern Brazil. Acta Genet Stat Med, 15, 177-89.

MONGALI, MA; EL-BAZ, M, CHAKROUN, D.; JEDIDI, If; DEBBABI, A (1991). Étude analytique des cas de diabètes infantiles dans un service de pédiatrie de Tunis ». Annal pédiatriques, vol. 38, no 9, pp. 623-626.

Mortad Nedjlaà et al., (2013). Etude Bio-Anthropologique des mariages consanguins et liens de parenté dans la population du littoral (Msirda) dans 
l'extrême Ouest Algérien, Thèse pour l'obtention du diplôme de Doctorat, pp 106-107-108-112.

Moussouni, A., (2011). Etude Anthropo- biologique de la consanguinité sur les paramètres de fitness et de morbidité dans la population de Sabra dans l'Ouest Algérien. Etude comparative dans le bassin Méditerranéen. Thèse pour l'obtention du diplôme de doctorat en anthropologie p 50 77,78.

OMS. (1993). La prévention de la cécité chez l'enfant, France. Oraganisation Mondiale de la Santé .

Rittler, M., Liascovich, R., Lopez-Camelo, J., \& Castilla, E. (2001). Parental consanguinity in specific types of congenital anomalies. Am J Med Genet , 102, 36-43.

Rudan I., Rudan D., Campbell H., Carothers A., Wright A., Smolej-Narancic N., Janicijevic B., Jin L., Chakraborty R., Debka R. et Rudan P., 2003a: Inbreeding and risk of late onset complex disease, Journal of Medical Genetics, 40, 925-932.

Saad F.A, Jauniaux E, 2002. Recurrent early pregnancy loss and consanguinity. Reprod Biomed Online, 5: 167-170.

Shami S.A, Schmitt LH, Bittles A.H., 1989. Consanguinity related prenatal and postnatal mortality of the populations of seven Pakistani Punjab cities. J Med Genet; 26: 267-271.

Sidi-Yakhlef, A., et al., (2012). Approche Anthropo-biologique de la consanguinité sur les paramètres de fitness et de morbidité dans la population de Oulhaça dans l'Ouest Algérien. Étude comparative a l'échelle du bassin méditerranéen. Thèse pour l'obtention du diplôme de Doctorat, p -83.

Solignac M et al.,1995, G.n.tique et .volution. Hermann.

Talbi J. et al. (2007), Etude de la structure génétique de quatre populations marocaines dans la région de Doukkala: « Impact de la consanguinité sur la morbidité, la mortalité et la vie reproductive »; Thèse pour l'btention du Diplôme de Doctorat national, p 124-136.

Teebi A.S., 1994: Autosomal recessive disorders among Arabs: An overview from Kuwait. Journal of Medical Genetics, 31, 224-233.Human genetic 1994, 58(4), 321-329.

Tuncbilek E., Koc I., Consanguineous marriage in Turkey and its impacts on fertility and mortality. Annals of

Wong S.S., Anokute C.C, The effect of consanguinity on pregnancy outcome in Saudi Arabic. Journal of the Royal Society of health 1990, 110(4), 146-147.

Yaqoob, M., Cnattingius, S., \& al. (1998). Risk Factor for mortality in young children living under various social economic conditions in Lahore, Pakistan : with particular reference to inbreeding. Clin Genet , 54, 426-434.

Van Der Berghe P.L, 1983. Human inbreeding avoïdance : culture in nature. The Behavioral and Brain Sciences 6: 91-123 : USA. 
Verma I.C., Prema A., Puri R.K., Health effects of consanguinity in Pondicherry. Indien pediatrics 1992, 29(6), 685-692.

Vogel F. et Motulsky AG., 1997: Human Genetics, 3rd edition. Berlin: Springer Verlag. 